\title{
Peignes de fréquences femtosecondes pour la mesure des fréquences optiques
}

\section{Femtosecond frequency combs for optical frequencies metrology}

\author{
Yann LE COQ ${ }^{1}$, Rodolphe LE TARGAT ${ }^{1}$, Wei ZHANG $^{1}$, Adil HABOUCHA ${ }^{1}$, Jacques MILLO ${ }^{1}$, \\ Daniele NICOLODI ${ }^{1}$, Zenyu XU ${ }^{1}$, Tang $\mathrm{LI}^{1}$, Rodolphe BOUDOT ${ }^{2}$, Yann KERSALÉ ${ }^{2}$, Marc FISCHER ${ }^{3}$, \\ Ronald HOLZWARTH ${ }^{3}$, Signe SEIDELIN ${ }^{4}$, Michel ABGRALL ${ }^{1}$, Jocelyne GUÉNA ${ }^{1}$, Andre LUITEN ${ }^{5}$, \\ Michel LOURS ${ }^{1}$, André CLAIRON ${ }^{1}$, Giorgio SANTARELLI ${ }^{1}$ et Sébastien BIZE ${ }^{1}$ \\ 1 LNE-SYRTE, Observatoire de Paris/CNRS/UPMC, 61 Avenue de l'Observatoire, 75014 Paris, France, yann.lecoq@ obspm.fr. \\ 2 FEMTO-ST Institute, CNRS/ENSMM, 32 Avenue de l'Observatoire, 25044 Besançon Cedex, France. \\ 3 MenloSystems GmBH, Am Klopferspitz 19a, 82152 Martinsried, Allemagne. \\ 4 Institut Néel, CNRS/Université Joseph Fourier, BP 166, 25 Rue des Martyrs, 38042 Grenoble Cedex 9, France. \\ 5 School of Physics, University of Western Australia, 6009 Crawley, Australie.
}

\section{Résumé}

L'avènement des peignes de fréquences, au début des années 2000 a révolutionné la métrologie des fréquences optiques (i.e. quelques centaines de térahertz), et a stimulé le développement d'une nouvelle génération d'horloges atomiques, référencées sur des transitions optiques. Ces peignes, réalisés avec des lasers à impulsions femtosecondes, sont aujourd'hui le moyen le plus efficace et fiable pour réaliser des comparaisons de fréquences entre une horloge du domaine optique et des étalons primaires du domaine micro-onde, ainsi qu'entre deux horloges optiques fonctionnant à des fréquences différentes. Nous présentons dans cet article divers travaux ayant été effectués au LNE-SYRTE pour caractériser ces dispositifs et les amener à un niveau de performance tel qu'ils ne limitent pas les mesures effectuées à partir des horloges du laboratoire.

MOTS CLÉS : MÉTROLOGIE TEMPS FRÉQUENCES, LASER FEMTOSECONDE, PEIGNE DE FRÉQUENCES, MÉTROLOGIE DES FRÉQUENCES OPTIQUES, MICRO-ONDES À BAS BRUIT DE PHASE.

\footnotetext{
Abstract

The advent of frequency combs, in the early 2000s, was a revolution for the metrology of optical frequencies (a few hundreds of terahertz), and stimulated the development of a new generation of atomic clocks, this time referenced to an optical frequency. These combs, based on lasers delivering femtosecond long pulses, are today the most efficient
}

and reliable way to compare an optical clock to microwave primary frequency standards, or directly two optical clocks operated at different frequencies. In this paper we report on the work carried out at LNESYRTE to characterize these devices. We describe the steps necessary to have the combs performing such that they do not limit the frequency comparison between the various clocks of our laboratory.

KEYWORDS: TIME AND FREQUENCY METROLOGY, FEMTOSECOND LASER, FREQUENCY COMB, OPTICAL FREQUENCY METROLOGY, LOW-PHASE NOISE MICROWAVE.

\section{Introduction}

Les horloges atomiques fonctionnant dans le domaine micro-onde (i.e. à une fréquence de quelques gigahertz), sur lesquelles se basent les réalisations de l'unité SI du temps, la seconde, et le temps atomique international [1], atteignent aujourd'hui leur limite, et les systèmes fonctionnant dans le domaine optique sont, à terme, amenés à les remplacer. Ces derniers dispositifs fonctionnent en interrogeant une transition atomique hyper étroite à l'aide d'un laser de très haute stabilité et pureté spectrale. En asservissant la fréquence du laser de sorte qu'il reste à la résonance de la transition atomique sondée, on réalise ainsi un étalon de fréquence dans le domaine optique (i.e. à une 
fréquence de quelques centaines de térahertz). La stabilité en fréquence de ces étalons optiques (quelques $10^{-15}$ à $1 \mathrm{~s}$ actuellement) surpasse largement celle des étalons de fréquences micro-ondes (à base de césium ou de rubidium), ce qui constitue une très forte motivation pour leur développement. Par ailleurs, une meilleure stabilité est également un moyen de caractériser les biais systématiques pouvant impacter un étalon de fréquence beaucoup plus rapidement et précisément. En termes d'exactitude, de nombreux effets sont insensibles à la fréquence de référence (effet Zeeman, effet de densité par exemple), et deviennent donc beaucoup plus petits en valeur relative, lorsqu'ils sont rapportés à une fréquence optique plutôt que micro-onde. Seuls les effets liés au mouvement (effet Doppler, effet de recul) dépendent de la fréquence, mais ce point particulier a été résolu pour les horloges optiques en confinant les particules soit dans un réseau optique (atomes neutres), soit dans un piège à ions (particules chargées).

L'avantage lié à l'utilisation des étalons de fréquences optiques est donc particulièrement clair. L'inconvénient est que le signal fournit par ce type d'horloge est un champ électromagnétique optique dont la fréquence d'oscillation est de plusieurs centaines de térahertz : aucun système électronique n'est suffisamment rapide pour la mesurer ou la comparer à d'autres étalons de fréquence, qu'ils soient optiques (à d'autres longueurs d'ondes) ou micro-ondes.

Avant le développement du peigne de fréquences, la seule solution pour effectuer des mesures de fréquences optiques passait par des chaînes de multiplication de fréquences successives extrêmement complexes, impliquant des dizaines de lasers, et très lourdes à mettre en œuvre, à tel point que seul un petit nombre de laboratoires dans le monde possédait une telle technologie. Les peignes de fréquences, générés par des lasers femtosecondes autoréférencés [2-8], permettent aujourd'hui de réaliser une telle mesure avec un dispositif simple, très compact (surface d'environ $1 \mathrm{~m}^{2}$ ), fiable, et désormais disponible commercialement.

Dans cet article, nous décrivons les travaux que nous avons menés au LNE-SYRTE depuis plusieurs années visant à développer plusieurs types de peignes de fréquences, qui soient aussi performants et fiables que possible. Ces dispositifs permettent à la fois de mesurer des fréquences optiques de façon absolue, en référençant le peigne sur les horloges micro-ondes du laboratoire, et de comparer différentes fréquences optiques entre elles.

\section{Principe d'un peigne de fréquences optiques}

La lumière émise par un laser femtoseconde à mode bloqué se compose, dans le domaine fréquentiel, d'une série de modes régulièrement espacés en fréquence et cohérents en phase les uns avec les autres. La fréquence optique de chaque mode, $v_{N}$, suit ainsi la relation : $v_{N}=N f_{\text {rep }}+f_{0}$, où $f_{\text {rep }}$ est la cadence de répétition (radio- ou basse fréquence) des impulsions laser, $N$ est un grand nombre entier, typiquement $10^{6}$, et $f_{0}$ est une

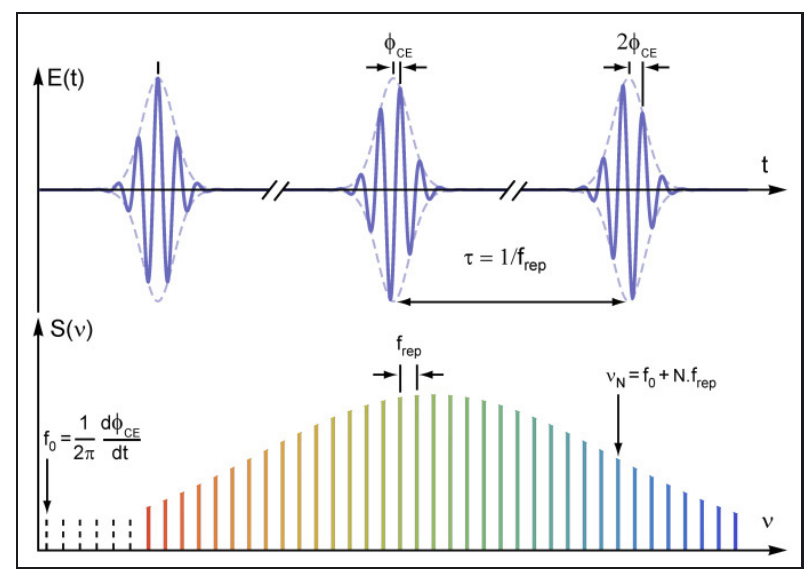

Fig. 1. - Correspondance entre les domaines temporel (impulsions successives) et fréquentiel (modes régulièrement espacés) pour le champ électrique issu d'un laser femtoseconde à modes bloqués. Le déphasage entre l'enveloppe et la porteuse suivant deux impulsions successives correspond, dans le domaine spectral, à un décalage global des dents d'une fréquence $f_{0}$.

fréquence de décalage globale appelée «fréquence de décalage entre l'enveloppe et la porteuse » (carrier-envelop offset frequency en anglais). Ce dernier terme est lié à la différence entre la vitesse de phase et la vitesse de groupe au sein de l'oscillateur laser et correspond, dans le domaine temporel, au fait que le champ électrique entre deux impulsions successives n'est pas strictement identique. Il présente un glissement de phase, par rapport à l'enveloppe temporelle des impulsions (fig. 1).

En mélangeant, sur une lame partiellement réfléchissante, les champs électriques issus d'un laser femtoseconde et d'un laser continu dont on veut connaître la fréquence optique absolue $v_{\mathrm{CW}}$, et en détectant le résultat avec une photodiode rapide (de bande passante $f_{\mathrm{BW}}$ ), on obtient un signal radiofréquence dont le spectre se compose d'une part d'harmoniques de la cadence de répétition $f_{\text {rep }}$, et d'autre part des différents battements optiques de fréquences $f_{\mathrm{b}, N}=\left|v_{\mathrm{CW}}-N f_{\text {rep }}-f_{0}\right|$, avec des valeurs de l'entier $N$ telles que $f_{\mathrm{b}, N}<f_{\mathrm{BW}}$. On peut alors facilement isoler de ce signal complexe, à l'aide d'un filtre radiofréquence passe-bande suffisamment étroit, l'une de ces composantes $f_{\mathrm{b}, N_{\mathrm{CW}}}$ qui fournit ainsi «l'écart en fréquence » du laser continu à mesurer avec l'un des modes du laser femtoseconde référencé par l'entier $N_{\mathrm{CW}}$.

De même, à l'aide d'un autre filtre passe-bande, on peut isoler l'une des harmoniques de la cadence de répétition $f_{\text {rep }}$; ce qui donne accès à cette valeur. En pratique, il est cependant fréquent de séparer physiquement les fonctions de mesure de $f_{\mathrm{b}, N_{\mathrm{CW}}}$ et $f_{\text {rep }}$ sur deux photodiodes différentes, afin d'optimiser les conditions de détection pour ces deux signaux séparément. Par exemple, l'optimisation du rapport signal à bruit du battement $f_{\mathrm{b}, N_{\mathrm{CW}}}$ nécessite en général de restreindre le spectre du laser femtoseconde illuminant la photodiode à un petit intervalle optique autour de la fréquence $v_{\mathrm{CW}}$. En effet, l'utilisation de l'ensemble du spectre du laser femtoseconde conduirait à une multitude de composantes de 
fréquences $f_{\mathrm{b}, N}$ supérieures à $f_{\mathrm{BW}}$ et produirait du bruit de type «bruit de grenaille» sans produire de signal utile. Limiter, dans la mesure du possible, ce bruit de grenaille ajouté, en restreignant le spectre à son domaine « utile » (avec un filtre interférentiel optique par exemple, de largeur spectrale de l'ordre ou inférieure à $1 \mathrm{~nm}$ environ) permet de gagner en rapport signal à bruit de détection de $f_{\mathrm{b}, N_{\mathrm{CW}}}$. Par contraste, la détection de $f_{\text {rep }}$ a tout avantage à utiliser au maximum la puissance optique disponible (dans la limite de la saturation de la photodiode) afin de maximiser le rapport signal à bruit de la détection. En outre, dans ce dernier cas, la partie 5 de l'article présente comment certains régimes de fonctionnement très particuliers permettent d'améliorer considérablement le bruit résiduel de mesure de $f_{\text {rep }}$, et ainsi d'utiliser par exemple le signal de détection obtenu comme source de microonde ultrabas bruit. Ces solutions ne peuvent être mises en place de façon fiable uniquement lorsqu'une photodiode est dédiée à la seule détection de $f_{\text {rep }}$ (ou de ses harmoniques).

Si l'on suppose que l'entier $N_{\mathrm{CW}}$ est connu ou du moins facile à déterminer (par exemple si l'on connaît déjà la fréquence $v_{\mathrm{CW}}$ à mesurer avec une exactitude meilleure que le taux de répétition $f_{\text {rep }}$ ), il reste uniquement la fréquence $f_{0}$ à déterminer pour contraindre l'ensemble du problème, et ainsi connaître absolument la fréquence optique $v_{\mathrm{CW}}$ à mesurer. Cette détermination de $f_{0}$ se fait par la technique de l'autoréférencement, qui présuppose que le laser femtoseconde ait un spectre couvrant au moins une octave (d'une fréquence quelconque $f$ à sa fréquence double $2 f$ ). En pratique, très peu de lasers possèdent cette propriété (seuls les lasers titane-saphir à impulsions ultracourtes de 3 fs à 5 fs environ présentent un spectre aussi étendu «naturellement»). Néanmoins, l'utilisation d'une fibre optique à haute non-linéarité permet, pour de nombreux systèmes, d'étendre le spectre jusqu'à la largeur nécessaire, tout en conservant les propriétés de cohérence nécessaires au fonctionnement du dispositif. Ces fibres optiques à haute non-linéarité sont ainsi conçues pour présenter une dispersion chromatique quasi nulle autour de la longueur d'onde centrale du laser femtoseconde, afin que les impulsions puissent s'y propager en restant très courtes et donc y conservent une forte intensité crête, gage de forts effets non-linéaires. Par mélanges à quatre ondes (éventuellement dégénérés) successifs entre les différents modes composant le spectre du laser femtoseconde, la fibre élargit ainsi progressivement le spectre jusqu'à atteindre un rapport de fréquences optiques supérieur ou égal à 2 entre ses deux extrémités, nécessaire pour la méthode d'autoréférencement. Ainsi il apparaît dans le spectre une composante de fréquence optique $v_{N}=N f_{\text {rep }}+f_{0}$ et une de fréquence $v_{2 N}=2 N f_{\text {rep }}+f_{0}$ à l'autre extrémité du spectre. En doublant la fréquence de la composante $v_{N}$ à l'aide d'un cristal non linéaire, et en produisant le signal de battement optique entre cette composante doublée, de fréquence $2 v_{\mathrm{CW}}$ et la composante de fréquence $v_{2 N}$ sur une photodiode rapide, on obtient ainsi un signal à la fréquence $\mid 2 N f_{\text {rep }}+2 f_{0}-2 N f_{\text {rep }}-$ $f_{0}|=| f_{0} \mid$ (voir fig. 2). Le doublement de fréquence agit sur plusieurs raies optiques $v_{N}$ simultanément, le signal

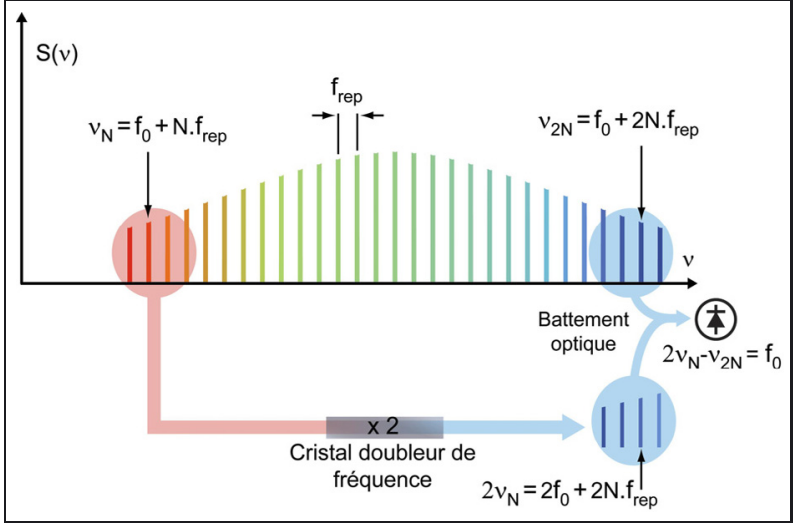

Fig. 2. - Schéma de principe de l'auto-référencement par la méthode $f-2 f$. Le battement optique entre l'extrémité «rouge » du peigne de fréquences doublée et l'extrémité «bleue» fournit un signal présentant une composante à la fréquence $f_{0}$, qu'il est facile d'isoler à l'aide d'un filtre radiofréquence passe-bande étroit. Noter que plusieurs paires de modes doivent combiner leurs signaux de façon cohérente pour obtenir un signal à fréquence $f_{0}$ de grand rapport signal à bruit.

obtenu présente donc également des harmoniques de la cadence de répétition $f_{\text {rep }}$, mais aussi des composantes de fréquences $\left|M f_{\text {rep }} \pm f_{0}\right|$ où $M$ est un entier. Il est néanmoins facile de supprimer ces composantes supplémentaires à l'aide de filtres passe-bande radiofréquences suffisamment étroits. Précisons également que la participation d'un grand nombre de paires de modes de fréquences $2 v_{N}$ et $v_{2 N}$ au signal est en réalité tout à fait favorable puisque plusieurs paires verront leur battement optique s'additionner de façon cohérente afin de produire une composante de fréquence $f_{0}$ d'amplitude bien supérieure à ce que donnerait une unique paire de modes. La puissance optique par mode étant typiquement de l'ordre de quelques picowatts, on voit bien là l'intérêt de ce processus de sommation cohérente. La sommation cohérente des signaux de fréquence $f_{0}$ issus de paires de modes $\left(2 v_{N}, v_{2 N}\right)$ ne peut néanmoins se produire que si chaque paire produit un signal de phase identique à celles des autres, ce qui nécessite un ajustement fin de la dispersion globale des systèmes optiques réalisant cet interféromètre $f-2 f$ (longueur optique effective traversée par les composantes de fréquences $v_{2 N}$ et celle traversée par les composantes de fréquence $v_{N}$ avant, pendant et après doublage de fréquence). Dans le domaine temporel, cette condition d'accord de phase est en fait équivalente à s'assurer que la partie de l'impulsion qui a été doublée en fréquence et celle qui ne l'a pas été arrivent simultanément sur la photodiode de détection du battement. Cette condition peut, par exemple, être simplement réalisée à l'aide d'une ligne à retard ajustable n'agissant que sur une partie du spectre optique (avant doublage de fréquence) ou bien par des éléments de dispersion fixe (lame de verre ou miroirs à dispersion négative) $a d$ hoc, ajoutés dans le trajet de propagation du faisceau.

L'ensemble des signaux radiofréquences $f_{\mathrm{b}, N_{\mathrm{CW}}}, f_{\text {rep }}$ et $f_{0}$ fournit ainsi l'intégralité des paramètres déterminant le système, et l'on a ainsi un dispositif permettant de comparer les fréquences optiques aux fréquences 


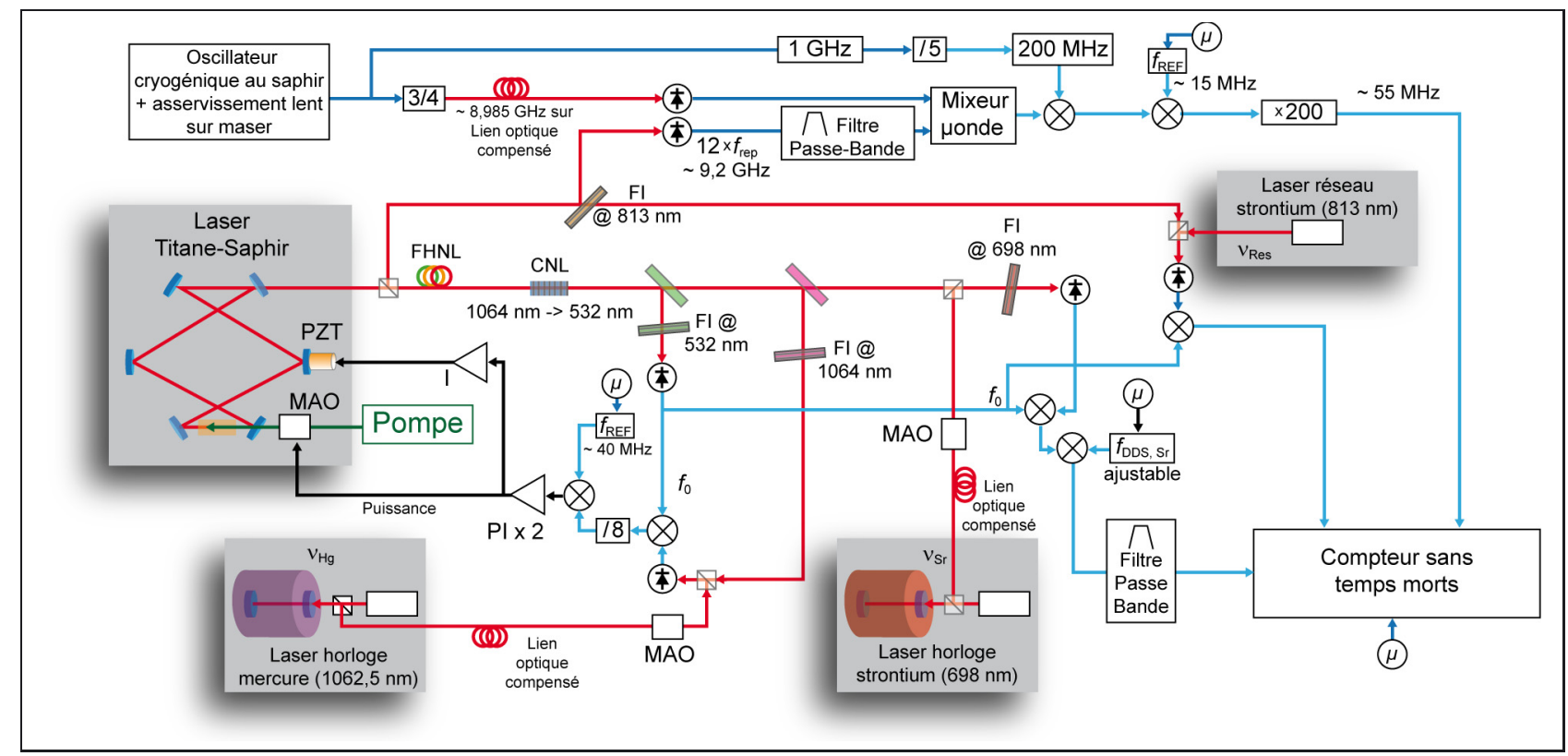

Fig. 3. - Architecture du peigne de fréquences titane-saphir du LNE-SYRTE : l'oscillateur femtoseconde est verrouillé en phase sur le laser à 1,06 $\mu \mathrm{m}$, lui-même asservi sur une cavité ultra-stable. La boucle de verrouillage est fermée grâce à une rétroaction rapide (PI : Proportionnel + Intégrateur) sur le modulateur acousto-optique (MAO) permettant de moduler la puissance de pompe du laser, et lente (I : Intégrateurs supplémentaires) sur la cale piézoélectrique (PZT) supportant un miroir de la cavité. Une fibre à cristaux photonique permet l'obtention d'un spectre optique couvrant plus d'une octave permettant l'obtention du signal d'auto-référencement $f-2 f$, ainsi que les différents battements optiques avec les laser à 1,06 $\mu \mathrm{m}$ (laser horloge de l'étalon de fréquence optique à mercure neutre), $698 \mathrm{~nm}$ (laser horloge de l'étalon de fréquence optique à strontium neutre) et $813 \mathrm{~nm}$ (laser piège de l'étalon de fréquence à strontium neutre). En version électronique, les lignes rouges correspondent aux faisceaux optiques, les lignes bleues aux signaux RF ou micro-ondes, les lignes noires aux signaux (quasi) continus. Les signes « $\mu$ » indiquent un référencement aux étalons primaires micro-ondes du laboratoire. FI : filtre interférentiel (passe-bande sur, typiquement $0,1 \mathrm{~nm}$ à $1 \mathrm{~nm}$ ); MAO : modulateur acousto-optique ; PZT : céramique piezoélectrique ; CNL : cristal non linéaire pour le doublage de fréquence optique.

micro-ondes, plus traditionnelles en métrologie tempsfréquence. En outre, en disposant de plusieurs battements entre le peigne et différents lasers continus (à différentes longueurs d'ondes), $f_{\mathrm{b}, N_{\mathrm{CW}}^{(1)}}, f_{\mathrm{b}, N_{\mathrm{CW}}^{(2)}}, f_{\mathrm{b}, N_{\mathrm{CW}}^{(3)}}$, etc. il est possible de comparer simultanément plusieurs fréquences optiques, sans nécessairement passer par l'intermédiaire des références micro-ondes.

Ces mesures présupposent néanmoins que les indices des modes $N_{\mathrm{CW}}^{(\mathrm{i})}$ soient connus. Le moyen le plus simple pour effectuer cette détermination est que la fréquence optique à mesurer soit préalablement connue avec une exactitude supérieure à la cadence de répétition du peigne de fréquences $f_{\text {rep }}$. En effet, dans ce cas, il est facile de situer la dent du peigne la plus proche de $v_{\mathrm{CW}}$. C'est le cas en particulier pour les lasers dits «d'horloge » du LNE-SYRTE, référencés sur des transitions atomiques dont la fréquence a été évaluée avec une grande précision au laboratoire. Dans le cas où cette connaissance a priori n'est pas suffisante, il est tout de même relativement aisé de déterminer l'entier $N_{\mathrm{CW}}$, en effectuant plusieurs mesures successives à plusieurs cadences de répétition du peigne $f_{\text {rep }}$ différentes, et en imposant que les résultats de ces mesures doivent être identiques (i.e. en supposant que la fréquence $v_{\mathrm{CW}}$ n'évolue que de façon négligeable entre les mesures successives). Si l'on possède plusieurs peignes de fréquences fonctionnels, on peut même envisager de réaliser les mesures à différentes cadences de répétition $f_{\text {rep }}$ simultanément (voire de façon synchrone) [9], ce qui relaxe la nécessité de faibles fluctuations de $v_{\mathrm{CW}}$ et de précision de mesure en général.

\section{Peignes de fréquences optiques au LNE-SYRTE}

Le LNE-SYRTE possède plusieurs peignes de fréquences optiques basés sur deux technologies différentes. Les peignes basés sur des lasers titane-saphir sont apparus dès le début 2000, et ont permis la mesure de nombreuses fréquences dans le domaine visible et infrarouge proche (fig. 3). Leur fonctionnement continu est néanmoins limité à quelques heures généralement à cause d'instabilités fondamentales inhérentes à ce type de laser. Une nouvelle génération de peignes basés sur des lasers fibrés est apparue au cours des années 2000 et des développements effectués en collaboration entre le LNE-SYRTE et un partenaire industriel (MenloSytems $\mathrm{GmBH})$ ont permis d'amener ces dispositifs à l'état de l'art.

\subsection{Système à base de titane-saphir}

\subsubsection{Description du laser}

La première génération de systèmes utilise comme oscillateur laser un cristal en saphir dopé avec des ions 
titane et placé dans une cavité en espace libre (fig. 3). Ce dispositif est pompé à $532 \mathrm{~nm}$ par un laser commercial Verdi de $8 \mathrm{~W}$, basé sur le doublage intra-cavité d'un laser Nd:YAG. Le fonctionnement pulsé en mode bloqué est assuré par l'effet Kerr au sein du cristal titane-saphir. La cadence de répétition est proche de $767 \mathrm{MHz}$, finement ajustable par un actuateur piézoélectrique supportant l'un des miroirs de la cavité. Le faisceau pompe traverse, avant d'atteindre l'oscillateur, un modulateur acousto-optique (utilisé dans l'ordre 0), qui permet d'ajuster finement la puissance de pompe effective sur une ampltitude maximale de $10 \%$, et ce de façon très rapide (une bande passante de $400 \mathrm{kHz}$, limitée à la fois par le temps de réponse de l'acousto-optique et celui du milieu de gain titane-saphir est typiquement accessible). Cette possibilité d'ajustement agit à la fois sur la fréquence $f_{0}$ et sur la cadence de répétition $f_{\text {rep }}$, par modification de l'indice et de la dispersion du cristal titane-saphir. Un coin de verre motorisé permet par ailleurs un réglage grossier de la fréquence $f_{0}$, en ajoutant ou retranchant une épaisseur de verre dispersif dans la cavité laser. Les impulsions produites, typiquement de 30 fs dans notre cas, sont envoyées en parallèle sur un photodétecteur GaAs rapide (pour la détection des harmoniques de la cadence de répétition), et sur une fibre à cristaux photoniques non-linéaire de $20 \mathrm{~cm}$ de long, qui permet de réaliser à sa sortie un quasicontinuum couvrant plus d'un octave du spectre optique (de 520 nm à 1100 nm environ). Différents éléments optiques en espace libre permettent alors de produire, à partir de ce spectre élargi, le signal d'auto-référencement $f_{0}$ ainsi que les battements optiques entre le peigne et les lasers continus dont il est nécessaire de mesurer la fréquence à l'heure actuelle au laboratoire : lasers d'horloge $(698 \mathrm{~nm})$ et de de piégeage $(813 \mathrm{~nm})$ pour les horloges au strontium, laser d'horloge (1 $062,5 \mathrm{~nm})$ pour l'horloge au mercure, laser stabilisé sur l'iode (1064 nm).

\subsubsection{Référencement du peigne sur un oscillateur externe à $1062,5 \mathrm{~nm}$, régime de raie étroite}

Le signal de battement à $\lambda_{\mathrm{CW}}=1062,5 \mathrm{~nm}$ possède un très bon rapport signal à bruit $(>50 \mathrm{~dB}$ dans une bande de $500 \mathrm{kHz}$ ). Ceci est dû au fait que la fibre à cristaux photonique utilisée pour l'obtention d'une octave de spectre est optimisée pour un signal $f-2 f$ maximal, et donc une puissance optique par mode maximale vers $1,1 \mu \mathrm{m}$ (et vers $550 \mathrm{~nm}$ ). Par conséquent, la puissance optique par mode disponible à $1062,5 \mathrm{~nm}$ est elle aussi «naturellement» optimale, sans devoir trouver de compromis entre les différents signaux de battements issus du peigne après la fibre à cristaux photonique. Nous utilisons cette propriété pour asservir le peigne de fréquences directement sur le laser ultra-stable dont nous disposons à cette longueur d'onde. Ce battement est, comme tous les autres signaux de battements optiques obtenus, amplifié et mélangé à $f_{0}$. À l'issue de ce mélange, on obtient principalement deux composantes de fréquences $\left|v_{\mathrm{CW}}-N_{\mathrm{CW}} f_{\text {rep }}-f_{0}+f_{0}\right|$ et $\left|v_{\mathrm{CW}}-N_{\mathrm{CW}} f_{\text {rep }}-f_{0}-f_{0}\right|$, la première étant indépendante de $f_{0}$ et des fluctuations associées. On isole cette composante radiofréquence à l'aide d'un filtre passe bande, on la divise en fréquence par un facteur 8 (ce qui permet in fine de gagner en fiabilité en limitant grandement la probabilité d'observer des sauts de cycles quand on réalise une boucle d'asservissement de phase), et on la fait battre avec une référence $f_{\mathrm{REF}}$ de fréquence fixe issue d'un synthétiseur référencé par rapport aux étalons primaires du laboratoire. Après filtrage passe-bas, le signal d'erreur ainsi obtenu est traité dans un correcteur type proportionnel plus intégrateurs multiples qui agit, aux fréquences de Fourier rapides, sur la puissance de pompe (via l'ordre 0 de l'AOM à $532 \mathrm{~nm}$ ), et aux fréquences de Fourier lentes $(<1 \mathrm{kHz})$ sur l'actuateur piézoélectrique qui permet d'ajuster la longueur de la cavité laser. On réalise ainsi une boucle à verrouillage de phase, qui relie la cadence de répétition $f_{\text {rep }}$ du peigne et la fréquence $v_{C W}$ de la référence optique par la relation :

$$
\frac{\left|v_{\mathrm{CW}}-N_{\mathrm{CW}} f_{\mathrm{rep}}\right|}{8}=f_{\mathrm{REF}} \text {. }
$$

En pratique, on n'utilise pas la dent la plus proche de $v_{\mathrm{CW}}$, mais on se décale de 3 ou 4 dents afin d'obtenir un battement de l'ordre de $1 \mathrm{GHz}$ pour éliminer au maximum le bruit électronique. Grâce à cette boucle de verrouillage de phase, on connecte de façon cohérente deux mondes de fréquences séparés par plus de cinq ordres de grandeur (radiofréquence pour $f_{\text {rep }}$ et optique pour $v_{\mathrm{CW}}$ ), via un facteur de division entier $N_{\mathrm{CW}}$ et un décalage de fréquence fixe $f_{\mathrm{REF}}$.

Dans la mesure où $f_{0}$ est éliminé des battements, il n'est pas nécessaire d'avoir une connaissance très précise de cette quantité. On veille simplement à ne pas introduire de retards électroniques différentiels excessifs entre le battement optique et $f_{0}$ afin de ne pas s'exposer au bruit de haute fréquence résiduel du profil spectral de $f_{0}$. Une fois le battement optique affranchi de $f_{0}$, le signal résultant «utile » présente une pureté spectrale aussi bonne que celle du laser sur lequel le peigne de fréquences est asservi. En effet, la bande passante importante $(>400 \mathrm{kHz})$ de la boucle à verrouillage de phase permet d'atteindre le régime dit «de raie étroite» ou « ultra-stable » dans lequel le bruit de phase résiduel de l'asservissement est parfaitement négligeable, et où, donc, chacune des dents du peigne (virtuel, une fois le signal $f_{0}$ retranché) possède les mêmes performances spectrales que la référence optique sur laquelle celui-ci est asservi (voir fig. 5 pour un exemple de battement entre une dent d'un peigne dans ce régime et un second laser ultrastable, lui aussi de largeur de raie $<1 \mathrm{~Hz}$ ). Dans le cas où l'obtention de ce régime spécifique est impossible, la largeur de raie des dents du peigne une fois asservi est à peu près identique à celle du peigne libre (typiquement quelques dizaines à centaines de kilohertz dans le domaine optique), même si leur position centrale moyenne est bien fixe du fait de la boucle à verrouillage de phase.

Historiquement, nous avons également travaillé avec ce système en mode «multiplicatif », où l'on asservit la cadence de répétition $f_{\text {rep }}$ directement sur une référence radiofréquence $(\mathrm{RF})$ dérivée des horloges micro-ondes à l'état de l'art du laboratoire. Les fréquences optiques sont 


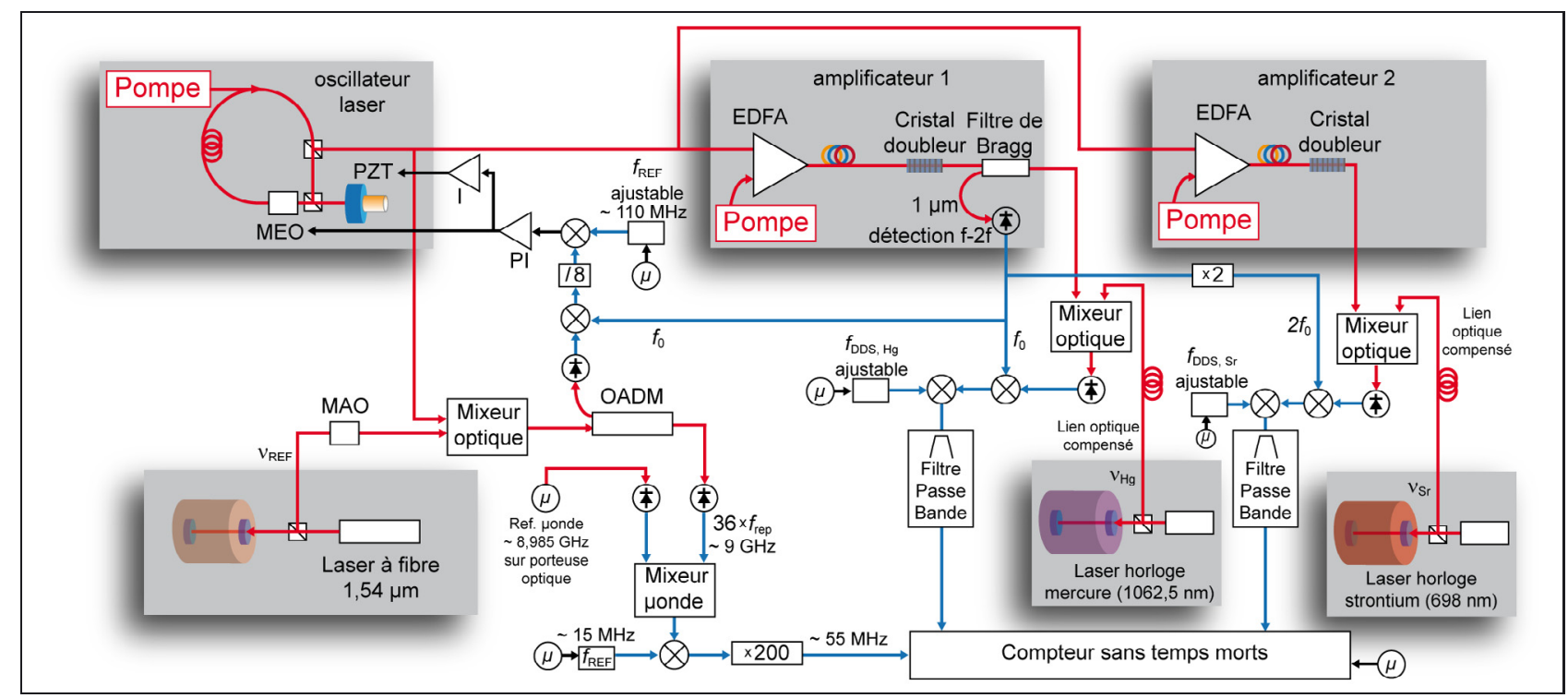

Fig. 4. - Architecture du peigne de fréquences fibré du LNE-SYRTE : l'oscillateur femtoseconde est verrouillé en phase sur le laser à $1,54 \mu \mathrm{m}$, lui-même asservi sur une cavité ultra-stable dont les dérives de fréquence sont compensées par un modulateur acoustooptique (MAO). La boucle de verrouillage est fermée grâce à une rétroaction rapide (PI : Proportionnel + Intégrateur) sur le modulateur électro-optique (MEO) intra-cavité, et lente (I : Intégrateurs supplémentaires) sur la cale piézoélectrique (PZT) supportant un miroir de la cavité. L'oscillateur injecte deux amplificateurs optiques (EDFA), le premier étant dédié à l'obtention de la fréquence $f_{0}$ ainsi qu'à la mesure de la fréquence $v_{\mathrm{Hg}}$ du laser horloge au mercure, et le second à la mesure de la fréquence $v_{\mathrm{Sr}}$ du laser d'horloge au strontium. En version électronique, les lignes rouges correspondent aux faisceaux optiques, les lignes bleues aux signaux RF ou microondes, les lignes noires aux signaux (quasi) continus. Les signes « $\mu$ » indiquent un référencement aux étalons primaires micro-ondes du laboratoire.

alors toutes mesurées en formant le battement entre le laser à mesurer et la dent du peigne la plus proche et en comptant la fréquence du battement avec un compteur RF. Cette méthode, quoique nettement plus simple à mettre en œuvre, présente l'inconvénient de multiplier le bruit de la référence RF vers le domaine optique et tous les bruits électroniques attenants. Par opposition, la méthode utilisée aujourd'hui (en mode « diviseur») divise le bruit de la référence optique (et les bruits électroniques associés) pour produire une fréquence $\mathrm{RF} f_{\text {rep }}$ de très haute performance en terme de bruit de phase. Les mesures finales ont ainsi une stabilité bien supérieure.

\subsection{Système à base de fibre dopée à l'erbium}

\subsubsection{Description du laser}

La seconde génération de peigne de fréquences du LNE-SYRTE utilise une technologie presque entièrement fibrée, à base de laser à fibre dopée à l'erbium (fig. 4). Ces peignes de fréquences sont composés d'une base commerciale largement modifiée pour atteindre un niveau de performance qui constitue l'état de l'art mondial actuel. L'oscillateur à blocage de mode passif se base sur l'effet non-linéaire de polarisation, dans lequel un choix judicieux des orientations de lames de polarisation intracavité permet de favoriser le fonctionnement en mode pulsé. Sur une longueur de quelques centimètres, la cavité est en fait réalisée en espace libre, ce qui permet la présence de plusieurs étages de contrôle de la cadence de répétition : un ajustement grossier à l'aide d'une platine de translation motorisée, un ajustement plus fin avec une cale piézoélectrique contrôlant la position d'un des miroirs, et un réglage ultrafin et très rapide avec un cristal électro-optique. Un ajustement grossier de $f_{0}$ est également possible grâce à une lame de verre d'épaisseur variable motorisée intra-cavité. Cet oscillateur est pompé par des diodes de puissance fonctionnant à $980 \mathrm{~nm}$, ellesmêmes alimentées par des sources de courant constantes à bas bruit. Les lasers fournissent des impulsions de $300 \mathrm{fs}$ à une cadence de répétition de $250 \mathrm{MHz}$ et une puissance moyenne de $200 \mathrm{~mW}$.

Ces dispositifs ont l'avantage principal de présenter une fiabilité très largement accrue : un fonctionnement à mode bloqué en continu pendant 3 à 6 mois sans intervention humaine est assez courant aujourd'hui au prix de spécificités somme toute surmontables. Historiquement, ces lasers ont en effet un bruit (bruit d'amplitude ainsi que de phase de $f_{\text {rep }}$ comme de $f_{0}$ ) typiquement plus important que les lasers à cristal titane-saphir, essentiellement du fait des diodes lasers de puissance, relativement bruyantes, utilisées pour pomper ces dispositifs. Par ailleurs les durées de vie du milieu de gain à base d'erbium sont longues, ce qui rend difficile l'action rapide sur le laser à l'aide de la modulation de puissance de pompe. Une bande passante de rétroaction de l'ordre de quelques dizaines de kilohertz constitue ainsi une limite typique de ce qu'il est possible d'obtenir, ce qui se révèle normalement insuffisant pour atteindre le régime de raie étroite dont on a vu tous les avantages pour le laser titane-saphir. 
Nous avons contourné cette difficulté en insérant un cristal électro-optique au sein de la cavité laser qui produit un délai de groupe contrôlable par la tension appliquée à ses bornes et fournit donc un moyen d'agir très rapidement sur la cadence de répétition du laser femtoseconde. Nous avons ainsi pu obtenir des bandes passantes de rétroaction supérieures à $1,4 \mathrm{MHz}$, largement suffisantes pour atteindre le régime de raie étroite. Une étude poussée, en particulier du point de vue des couplages résiduels inévitables vers les autres degrés de liberté du laser, comme sa puissance ou sa fréquence $f_{0}$, a également été réalisée par notre équipe [10], et a démontré les grandes potentialités de ce type de système. Grâce au partenariat privilégié avec notre partenaire industriel (MenloSystems $\mathrm{GmBH})$, cette technologie est désormais disponible commercialement.

\subsubsection{Référencement du peigne sur un oscillateur externe à $1,54 \mu \mathrm{m}$, régime de raie étroite}

Le référencement du peigne à laser à fibre, ainsi que la chaîne de mesure de fréquences, sont décrits sur la figure 4. Une partie de la puissance optique de la cavité femtoseconde est filtrée par un OADM (Optical Add and Drop Module - filtre interférentiel très étroit issu de la technologie de télécommunications optiques) centré à 1,54 $\mu \mathrm{m}$. La composante filtrée est combinée avec un laser ultra-stable de même longueur d'onde (qui constitue l'oscillateur de référence pour les expériences de lien fibré optique) [11]. Le signal de battement obtenu, après filtrage, mélange au signal $f_{0}$, division en fréquence par un facteur 8 et mélange à une référence de fréquence fixe (générée par une DDS pour Direct Digital Synthesizer contrôlée par ordinateur) fournit le signal d'erreur du peigne de fréquence. Au moyen d'un correcteur proportionnel et de multiples intégrateurs en cascade, ce signal est appliqué sur le cristal électro-optique de l'oscillateur (fréquences de Fourier $>5 \mathrm{kHz}$ ) ainsi qu'à sa cale piézoélectrique (fréquences de Fourier $<5 \mathrm{kHz}$ ). Le peigne est alors verrouillé en phase sur la référence à 1,54 $\mu \mathrm{m}$ et atteint le régime de raie étroite. La partie du spectre transmise par le filtre OADM (environ $70 \mathrm{~mW}$ ) est intégralement envoyée sur un photodétecteur InGaAs rapide à haute linéarité (DSC40S HLPD, Discovery Semiconductors), qui détecte les différentes harmoniques de la cadence de répétition.

Une autre partie de la puissance de la cavité est envoyée dans un amplificateur à fibre dopé erbium suivi d'une fibre optique à haute non-linéarité qui étale le spectre sur une octave (de $1 \mu \mathrm{m}$ à $2 \mu \mathrm{m}$ environ). Un cristal doubleur PPLN en guide d'onde suivi d'une photodétection du battement entre la partie du spectre à $2 \mu \mathrm{m}$ doublé et celle à $1 \mu \mathrm{m}$ permet alors d'obtenir le signal $f_{0}$. Par ailleurs, une partie de la lumière obtenue vers $1 \mu \mathrm{m}$ est également utilisée pour générer le battement optique avec le laser ultra-stable à 1062,5 nm (horloge à mercure neutre du laboratoire). Enfin, une partie de la puissance de l'oscillateur femtoseconde est envoyée dans un second système amplificateur-fibre non linéaire, suivi d'un doubleur de fréquence qui replie une partie des dents du

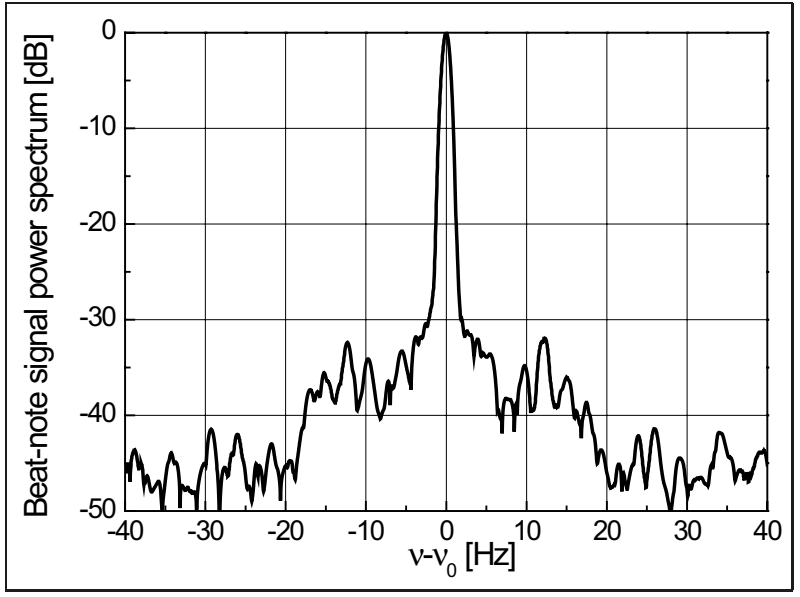

Fig. 5. - Exemple typique de battement optique entre l'un des modes d'un peigne de fréquence auto-référencé asservi, en régime de raie étroite, sur une référence ultra-stable (largeur de raie $<1 \mathrm{~Hz}$ ), et un second laser ultrastable, lui aussi de largeur de raie $<1 \mathrm{~Hz}$. Ce spectre est ici obtenu pour un peigne à fibre dopé erbium asservi sur une référence ultra-stable à $1542 \mathrm{~nm}$ et dont une des dents bats avec un laser ultra-stable à $1062,5 \mathrm{~nm}$ (signal de battement RF représenté ici sur la figure).

spectre autour de $698 \mathrm{~nm}$. Ce système permet de produire le battement optique avec le laser ultra-stable à $698 \mathrm{~nm}$ (horloge à strontium neutre du laboratoire). Après élimination de $f_{0}$, les signaux de battements à $1062,5 \mathrm{~nm}$ et $698 \mathrm{~nm}$ sont alors eux aussi à raie étroite (typiquement $<1 \mathrm{~Hz}$, voir la fig. 5 pour un exemple de ce type de battement) et sont donc aisément filtrables et amplifiables afin de voir leurs fréquences comptées par un système de compteur sans temps morts à multicanaux synchrones (type FXE de la compagnie Kramers-Klische).

\section{Mesures des fréquences optiques}

La stratégie de mesure de fréquences optiques est commune aux deux types de peignes. Ils sont tous deux référencés sur un laser optique très stable afin d'atteindre le régime de raie étroite, et la fréquence de ce laser est ramenée dans le domaine micro-onde par division afin de comparer le peigne aux étalons primaires de fréquence $\mathrm{du}$ laboratoire. Les différentes fréquences optiques sont mesurées par battement avec le peigne ainsi référencé.

\subsection{Mesures des paramètres des peignes}

\subsubsection{Taux de répétition}

Il est nécessaire de mesurer précisément et en permanence le taux de répétition $f_{\text {rep }}$ d'un peigne : ceci permet de déduire les valeurs absolues des fréquences optiques mesurées, ainsi que la fréquence de la référence optique sur laquelle le peigne est asservi et qui peut au besoin être disséminé comme signal ultra-stable de référence auprès d'autres laboratoires. On utilise le signal de détection des harmoniques de $f_{\text {rep }}$ par la photodiode InGaAs ou GaAs (selon le laser utilisé) rapide. 
Dans le cas du peigne titane-saphir, on isole la $12^{\mathrm{e}}$ harmonique de $f_{\text {rep }}$, de fréquence proche de 9,2 GHz, à l'aide d'un filtre passe-bande étroit, et on la mélange à une référence exactement égale à $9,185 \mathrm{GHz}$ issue des étalons primaires du laboratoire. Le battement résultant, de fréquence proche de $15 \mathrm{MHz}$, est alors mélangé à un signal lui aussi exact, mais de fréquence ajustable, afin d'obtenir une fréquence très proche de $275 \mathrm{kHz}$ (à moins de $1 \mathrm{~Hz}$ près). On la multiplie alors par exactement 200 pour aboutir à $55 \mathrm{MHz}$ environ, et on mesure cette fréquence finale à l'aide du compteur sans temps mort, qui possède plusieurs canaux synchrones (fig. 3). La dernière étape de multiplication est nécessaire afin d'utiliser le compteur au mieux de ses performances. En effet, celuici possède une résolution de $1 \mathrm{mHz}$ pour un temps de mesure de $1 \mathrm{~s}$, ce qui ne représenterait qu'une résolution d'environ $10^{-13}$ sur la mesure de l'harmonique $12 \times f_{\text {rep }}$, bien inférieure aux performances des étalons primaires disponibles au laboratoire. L'étape de multiplication permet de mesurer en $1 \mathrm{~s}$ le signal à $55 \mathrm{MHz}$ avec une résolution de $1 \mathrm{mHz}$, ce qui revient à effectuer la mesure du signal à $275 \mathrm{kHz}$ avec une résolution « virtuelle » de $5 \mu \mathrm{Hz}$ (pour $1 \mathrm{~s}$ de temps de mesure). Ramené à la porteuse de $9,2 \mathrm{GHz}$, ceci correspond à une résolution de $5,4 \times 10^{-16}$, compatible avec la stabilité de la référence à $9,185 \mathrm{GHz}$. Ce niveau de performance est meilleur que celui attendu des signaux distribués au laboratoire, et ne constitue donc pas une limite aux comparaisons optique-micro-onde au LNE-SYRTE.

Le principe de la détection de $f_{\text {rep }}$ est similaire pour les peignes fibrés, à l'exception du fait que la $36^{\mathrm{e}}$ harmonique est utilisée, et que le mélange se fait avec une référence exacte à $8,985 \mathrm{GHz}$, issue elle aussi de l'oscillateur micro-onde à saphir cryogénique du laboratoire, puis avec une source exacte et ajustable proche de $15 \mathrm{MHz}$ pour aboutir à une valeur très proche de $275 \mathrm{kHz}$. La résolution de mesure, après multiplication de ce signal pour aboutir à $55 \mathrm{MHz}$, est identique à celle du peigne titane-saphir.

\subsubsection{Indice des dents des peignes}

Afin de pouvoir mesurer les fréquences absolues, il faut aussi identifier l'indice $N$ du mode du peigne de fréquence qui sert à réaliser le battement optique avec le laser à mesurer, ainsi que les différents signes $( \pm 1)$ dans les formules arithmétiques donnant la fréquence optique $v_{\mathrm{CW}}$ en fonction de la fréquence du battement et de la fréquence de répétition $f_{\text {rep }}$. La fréquence de la porteuse optique $v_{\text {REF }}$ sur laquelle le peigne est verrouillé se déduit de la relation (1):

$$
v_{\mathrm{REF}}=N_{\mathrm{REF}} \times f_{\text {rep }}+8 \times \operatorname{sign}_{\mathrm{REF}} \times f_{\mathrm{REF}} .
$$

Le peigne ainsi référencé permet la mesure d'autres fréquences optiques $v_{\mathrm{CW}}^{(i)}$ en isolant leur battement avec la dent du peigne la plus proche, puis en le mélangeant de la même façon avec $f_{0}$ pour s'en affranchir :

$$
v_{\mathrm{CW}}^{(i)}=N_{\mathrm{CW}}^{(i)} f_{\text {rep }}+\operatorname{sign}^{(i)} \times f_{\text {beat }}^{(i)} .
$$

Trois approches complémentaires permettent une identification certaine des $N_{\mathrm{CW}}$ si les références optiques et micro-ondes à comparer sont suffisamment stables et/ou connue a priori.

Si la fréquence optique à mesurer est connue a priori avec une incertitude suffisamment petite devant le taux de répétition du peigne, la première méthode consiste juste en une identification directe. En effet, dans ce cas, un seul entier $N$, et un seul signe devant $f_{\text {beat }}^{(i)}$, vérifient l'équation (3), ce qui lève facilement l'indétermination. C'est le cas par exemple pour les lasers ultra-stables à $698 \mathrm{~nm}$ et à $1062,5 \mathrm{~nm}$, dont la fréquence ne change pas de plus que 1,5 MHz par an, ce qui reste plus de deux ordres de grandeur plus petit que les taux de répétition des peignes titane-saphir (de l'ordre de $767 \mathrm{MHz}$ ) et fibrés (environ $250 \mathrm{MHz}$ )

La dérive de fréquence ou les besoins expérimentaux d'accordabilité d'autres lasers du laboratoire peuvent ne pas être négligeables devant $f_{\text {rep }}$, il faut donc recourir à d'autres méthodes discriminantes en $N$. Précisons tout d'abord que la quantité $\operatorname{sigh}^{(i)}$ est en règle générale assez facilement déduite en relâchant la contrainte (1) (ouverture de la boucle à verrouillage de phase), puis en faisant varier $f_{\text {rep }}$ tout en observant le sens de variation de $f_{\text {beat }}^{(i)}$. Pour déterminer $N_{\mathrm{CW}}^{(i)}$, une seconde méthode consiste à effectuer deux comparaisons successives entre la fréquence optique à mesurer et la référence micro-onde en utilisant deux cadences de répétition différentes $f_{\text {rep }, 1}$ et $f_{\text {rep }, 2}$. On observe alors le battement de $v_{\mathrm{CW}}^{(i)}$ avec la dent $N_{\mathrm{CW}}^{(i)} \mathrm{du}$ peigne, puis la dent $N_{\mathrm{CW}}^{(i)}+M$, où $M$ est facilement déduit du défilement des raies lors du passage de $f_{\text {rep, } 1}$ à $f_{\text {rep }, 2}$. On a alors la relation :

$$
N=M \frac{f_{\text {rep }, 1}}{f_{\text {rep }, 2}-f_{\text {rep }, 1}}+\frac{\operatorname{sign}_{2}^{(i)} \times f_{\text {beat }, 2}^{(i)}-\operatorname{sign}_{1}^{(i)} \times f_{\text {beat }, 1}^{(i)}}{f_{\text {rep }, 2}-f_{\text {rep }, 1}} .
$$

L'incertitude sur $f_{\text {rep }}$, quelques $10^{-15}$ tout au plus, dus au bruit de la référence optique et de la micro-onde permettant l'évaluation de $f_{\text {rep }}$, mène à une incertitude sur $N$ bien inférieure à 1 dans (4). La dernière hypothèse sousjacente pour déduire une valeur sans ambiguïté de $N$ est que la dérive de la fréquence absolue $v_{\mathrm{CW}}^{(i)}$ entre les deux mesures doit être petite devant $f_{\text {rep }, 2}-f_{\text {rep, }, 1}$, condition aisément remplie en pratique au laboratoire, où les dérives des lasers à mesurer excèdent très rarement les quelques dizaines de hertz par seconde.

Enfin, on remarque en regardant les équations (2) et (3) que $N$ peut s'interpréter comme étant la sensibilité de la fréquence optique considérée a une variation de $f_{\text {rep }}$. La troisième procédure qui a été implémentée consiste donc à induire volontairement un changement de $f_{\text {rep }}$ en maintenant la boucle à verrouillage de phase fermée (condition (1) remplie). Si on prend exemple sur le laser de référence, on induit un changement de fréquence $\Delta$ (typiquement $+1,8 \mathrm{MHz}$ ) sur $f_{\mathrm{REF}}$ et on moyenne pendant $8 \mathrm{~s}$ pour obtenir la nouvelle valeur $f_{\text {rep },+}$ du taux de répétition. Au-delà de $8 \mathrm{~s}$, la mesure est limitée par 
le bruit thermique de la cavité du laser de référence (quelques $10^{-15}$ dans le cas $1,54 \mu \mathrm{m}$ et $4 \times 10^{-16}$ dans le cas $1062,5 \mathrm{~nm})$. Si $f_{\mathrm{REF}}$ et $f_{\text {rep }}$ varient dans le même sens, cela implique que $s i g n_{\mathrm{REF}}$ est égal à -1 , et +1 dans le cas opposé. Pour accroître la sensibilité, on renouvelle l'opération en induisant un décalage $-\Delta$ pour obtenir $f_{\text {rep,- }}$. L'entier $N_{\text {REF }}$ s'obtient alors par la relation suivante (ou, plus précisément, l'entier le plus proche de la valeur donnée par cette relation):

$$
N_{\mathrm{REF}}=\frac{\operatorname{sign}_{\mathrm{REF}} \times 2 \times 8 \times \Delta}{f_{\mathrm{rep},+}-f_{\mathrm{rep},-}} .
$$

Une stabilité au niveau de $10^{-15}$ sur $f_{\text {rep }}$ est suffisante pour avoir une incertitude petite devant 1 sur $N_{\mathrm{REF}}$. Ce calcul fait l'hypothèse que la dérive de la référence optique a été annulée. On peut facilement étendre le principe de cette mesure au cas où cette dérive est supposée linéaire de pente constante et où elle est calibrée en parallèle au cours de la mesure. L'avantage de cette approche est qu'il n'est pas utile d'interrompre la boucle à verrouillage de phase du peigne sur la référence optique, et que tout peut se faire de façon automatisée (comme c'est le cas pour les lasers peigne à fibre dopé erbium du LNESYRTE). Par contre, comme la valeur du décalage $\Delta$ de $f_{\text {REF }}$ qu'il est possible de réaliser sans provoquer d'ouverture de la boucle à verrouillage de phase est limitée à quelques megahertz, cette méthode nécessite d'être capable de faire des mesures optique-micro-onde à une résolution de quelques $10^{-15}$ en un temps sur lequel les dérives de fréquences restent prédictibles (dérive linéaire par exemple), soit typiquement quelques dizaines de secondes. La méthode n'est donc facile à mettre en œuvre que si l'on dispose d'un oscillateur de référence microonde ultra-stable extrêmement performant atteignant typiquement une stabilité de fréquences de quelques $10^{-15}$ sur ces échelles de temps (un oscillateur micro-onde à saphir cryogénique par exemple). Pour finir, précisons que cette dernière technique peut évidemment être également appliquée au calcul des nombres $N_{\mathrm{CW}}^{(i)}$, un décalage $\Delta f_{\text {rep }}$ induisant un changement de $f_{\text {beat }}^{(i)}$ dont le signe et l'amplitude permettent de déterminer $\operatorname{sign}^{(i)}$ et $N_{\mathrm{CW}}^{(i)}$.

\subsection{Mesures des fréquences optiques avec le peigne asservi}

Le logiciel d'acquisition de données du compteur sans temps mort que nous avons développé permet de traiter l'ensemble de ces informations (fréquences des différentes références, différents canaux du compteur) de façon à déduire les fréquences absolues des différents lasers $(1,54 \mu \mathrm{m}, 1062,5 \mathrm{~nm}, 698 \mathrm{~nm})$ par rapport aux étalons primaires micro-ondes du laboratoire.

\subsubsection{Laser de référence}

Dans le cas des peignes fibrés, la fréquence du laser à 1,54 $\mu \mathrm{m}$ est non seulement mesurée en permanence par le peigne, mais une boucle de rétroaction logicielle «molle » permet de corriger les dérives de fréquence avec un temps caractéristique de $100 \mathrm{~s}$. La correction s'effectue sur la fréquence d'un modulateur acousto-optique placé juste après le laser, de telle sorte que le faisceau utile a en pratique une fréquence aussi constante que possible. Ceci revêt une importance opérationnelle, afin de pouvoir utiliser les lasers à $1,54 \mu \mathrm{m}$ du LNE-SYRTE comme références distribuables à d'autres instituts. Dans la mesure où cette longueur d'onde se trouve dans l'une des fenêtres de prédilection des réseaux de télécommunications, ces lasers ont été choisis non seulement pour référencer les peignes fibrés, mais également pour servir de base à l'architecture des liens optiques fibrés REFIMEV+ dont le LNE-SYRTE est l'un des acteurs majeurs.

\subsubsection{Autres fréquences optiques}

La mesure simultanée des autres fréquences optiques est semblable à celle effectuée pour le laser de référence. Grâce à une fibre optique compensée contre les bruits à basse fréquence (vibrations, fluctuations de température), on amène physiquement un faisceau issu du laser à étudier à proximité du laser générant le peigne de fréquence, et on réalise leur battement. On corrige là encore ce battement de $f_{0}$, avant de le faire passer dans un filtre passebande. La fréquence du battement résultant $f_{\text {beat }}^{(i)}$ est mélangée à une référence exacte (DDS) dédiée afin de se ramener à une fréquence proche de $10 \mathrm{kHz}$. Ce battement final est filtré par un filtre passe-bande Butterworth d'ordre 4, d'une largeur à mi-hauteur de $1 \mathrm{kHz}$, et compté par un canal dédié du compteur sans temps mort décrit ci-dessus (fig. 3 et 4 ).

L'avantage considérable du régime de raie étroite apparaît clairement dans le contexte de mesure d'une seconde fréquence optique qui nous préoccupe ici. En effet, les quasi-continuums issus de fibres non-linéaires présentent de larges variations d'amplitude selon les domaines du spectre optique. Si des ajustements fins (puissance, polarisation...) permettent normalement d'optimiser les signaux à certaines longueurs d'ondes (on optimise en principe celles permettant d'obtenir le signal $f-2 f)$, il est quasi-impossible d'obtenir simultanément de fortes puissances optiques à toutes les longueurs d'onde d'intérêt métrologique. Pour le peigne basé sur le laser titane-saphir du laboratoire, le battement optique avec le signal à $698 \mathrm{~nm}$ présente ainsi, typiquement, un rapport signal à bruit (dans une bande de $500 \mathrm{kHz}$ ) entre $20 \mathrm{~dB}$ et $30 \mathrm{~dB}$ moins bon que celui avec le signal à $1062,5 \mathrm{~nm}$ (et que celui obtenu sur la mesure de $f_{0}$ ). Le régime de raie étroite permet de s'affranchir de ce problème car il autorise à filtrer très fortement le signal de battement (corrigé de $f_{0}$ ) sans que l'on perde de signal. Typiquement, le peigne de fréquences asservi sur le laser à $1062,5 \mathrm{~nm}$ du laboratoire présente une largeur de raie inférieure à $1 \mathrm{~Hz}$. Le laser à $698 \mathrm{~nm}$ présente lui aussi une pureté spectrale similaire, quoiqu'avec un rapport signal à bruit dégradé. En filtrant dans une bande de quelques centaines de hertz, on retrouve ainsi un rapport signal à bruit compatible avec une mesure de fréquence par le compteur dans les meilleures conditions. La stabilité relative des lasers du laboratoire à $1062,5 \mathrm{~nm}$ et $698 \mathrm{~nm}$ est ainsi mesurée aux environs de $10^{-15}$ à $1 \mathrm{~s}$ d'intégration. Cette performance est proche des stabilités intrinsèques attendues pour ces deux lasers l'un par rapport à 
l'autre (le bruit dû au peigne lui-même est négligeable). De la même façon que pour le laser à $1062,5 \mathrm{~nm}$, un traitement informatique en temps réel du flux de donnée du compteur permet d'enregistrer continûment la fréquence absolue du laser à 698 nm, référencé par rapport aux étalons primaires (micro-onde) du laboratoire. Si un fonctionnement en continu du peigne de fréquences titane-saphir du laboratoire asservi sur référence optique à $1062,5 \mathrm{~nm}$ pendant $8 \mathrm{~h}$ à $12 \mathrm{~h}$ est couramment obtenu, il est souhaitable, dans une perspective de déploiement opérationnel future des horloges optiques, de disposer de systèmes fonctionnant sans intervention humaine pendant plusieurs semaines, ce qui semble difficile à envisager pour les systèmes titane-saphir existants. Le déploiement et la généralisation actuellement en cours de l'emploi de peignes à fibres dopées à l'erbium s'inscrit ainsi dans ce contexte et plusieurs jours de mesures en continu sans intervention humaine avec de tels systèmes sont d'ores et déjà régulièrement répétés.

De nombreuses campagnes de mesure utilisant ces peignes de fréquences ont été et sont encore réalisées régulièrement au LNE-SYRTE. Sans rentrer dans une liste exhaustive, on citera en particulier les premières mesures de fréquence horloge du mercure neutre, la première réalisation d'une horloge à mercure neutre (avec une stabilité de quelques $10^{-15}$ à $1 \mathrm{~s}$ ) $[12,13]$, la caractérisation des horloges à strontium neutre du laboratoire contre les références primaires à un niveau limité par l'exactitude de celles-ci [14], ainsi que de fréquentes campagnes de mesure de références à 1,54 $\mu \mathrm{m}$ pour le lien optique longue distance, en collaboration avec le Laboratoire de physique des lasers à Villetaneuse [15]. Nos dispositifs sont également régulièrement utilisés en assistance au développement de divers projet du laboratoire : Lien optique en espace libre (projet MiniDOLL), laser stabilisé sur l'iode moléculaire (projet LASIC), voire pour des prestations de services à des industriels (Thales Alénia Space...)

\section{Génération de signaux micro-onde à très bas bruit de phase, application aux fontaines atomiques}

Au-delà des mesures «utilitaires » de fréquence absolue ou de stabilité effectuées pour les différents étalons de fréquences optiques du laboratoire, un domaine d'application des peignes de fréquences dans lequel nous avons tout particulièrement travaillé est la génération de signaux micro-ondes à très bas bruit de phase. L'objectif premier de cette technique est d'utiliser ces signaux comme oscillateur d'interrogation pour les fontaines atomiques du laboratoire qui ont besoin d'une très bonne référence micro-onde afin d'opérer à leur optimum de stabilité, définie par le bruit de projection quantique. À l'heure actuelle, les fontaines du laboratoire utilisent un système à saphir cryogénique comme oscillateur d'interrogation, système très performant mais lourd et coûteux à entretenir et utiliser. À terme, nous projetons de le remplacer par un système purement photonique constitué d'un peigne de fréquence asservi sur une référence optique ultra-stable (cavité Fabry-Pérot de très haute performance développée au laboratoire $[16,17])$. Le principe de fonctionnement d'une telle approche est le suivant : un peigne de fréquences asservi sur une référence optique génère (par photodétection du train d'impulsions) un signal micro-onde qui correspond à la division de la fréquence optique (quelques centaines de térahertz) par un facteur de quelques $10^{4}$ environ. Le bruit de phase du signal micro-onde est donc idéalement égal à celui de l'oscillateur optique moins $20 \times \log (A)$, où $A$ est le rapport entre la fréquence optique de référence et la fréquence micro-onde générée par photo-détection du train d'impulsions, soit un niveau potentiellement très prometteur, même si, bien sûr, le peigne de fréquences est en pratique imparfait et dégrade les performances par rapport à l'idéal «mathématique». Nous avons démontré qu'un tel système peut fournir un signal d'interrogation pour les fontaines atomiques du laboratoire à un niveau de performance tel que la stabilité de la comparaison est là aussi limitée par le bruit de projection quantique des fontaines, de façon équivalente au cas de l'utilisation de l'oscillateur à saphir cryogénique [18] (voir également [19]).

Afin d'explorer les limites ultimes de tels systèmes, nous avons effectués de nombreuses expériences « différentielles », c'est-à-dire en asservissant des peignes de fréquences (à base de fibres dopées à l'erbium) quasiidentiques sur une même référence optique ultra-stable et en comparant le bruit de phase relatif entre les deux signaux micro-ondes fournis par les deux systèmes [20]. Ce type d'expérience permet de caractériser le bruit résiduel ajouté par le peigne de fréquence au processus de division «idéal».

Pour résumer nos études, il apparaît que deux processus principaux limitent en premier lieu les performances obtenues. Pour les fréquences de Fourier faibles ( $<10 \mathrm{kHz}$ typiquement), les fluctuations d'amplitude du laser à impulsions femtosecondes générant le peigne de fréquences sont converties en fluctuation de phase lors $\mathrm{du}$ processus de photodétection du train d'impulsions qui produit le signal micro-onde. Pour les fréquences de Fourier plus élevées, et pour les lasers à fibre équipés d'un modulateur électro-optique intra cavité (et disposant donc d'une bande passante d'asservissement importante), une limite importante provient du rapport signal à bruit limité que l'un obtient en sortie de la photodiode InGaAs à une harmonique donnée de la cadence de répétition.

Le premier problème est celui de la conversion amplitude-phase, pour lequel nous avons pu démontrer tout l'intérêt d'un asservissement actif de la puissance [21,22], qui ne peut malheureusement être réalisé que dans une bande passante limitée (quelques dizaines de kilohertz). Mais une étude poussée du processus nous a également permis de démontrer l'existence de point de fonctionnement «magiques »: pour certaines énergies par impulsion, le coefficient de conversion amplitudephase est exactement nul [23] (fig. 6). L'emploi de ces points «magiques » présente un intérêt primordial pour la génération de signaux micro-ondes à bas bruit de phase, et nous nous employons aujourd' hui à rendre leur utilisation systématique dans les expériences. 


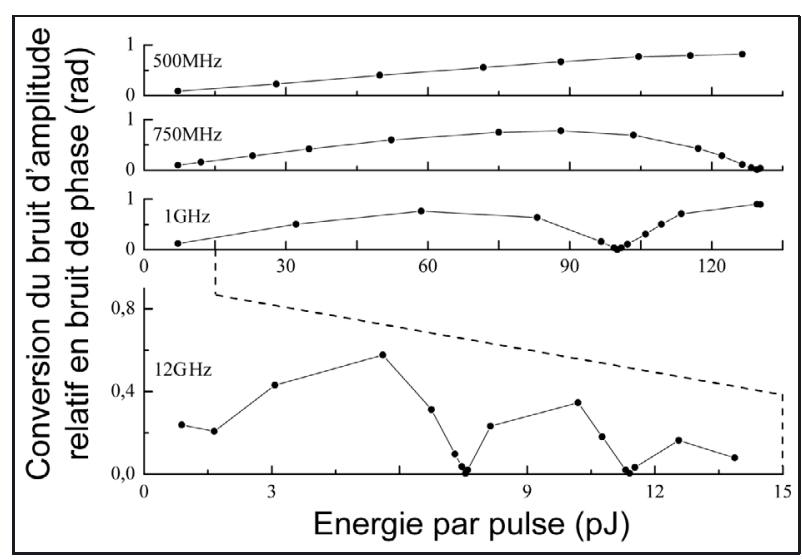

Fig. 6. - Valeur mesurée du coefficient de conversion entre le bruit d'amplitude optique et le bruit de phase micro-onde, en fonction de l'énergie par impulsion du laser femtoseconde. Le résultat est montré pour les harmoniques 2, 3, 4 et 48 (resp. $500 \mathrm{MHz}, 750 \mathrm{MHz}, 1 \mathrm{GHz}$ et $12 \mathrm{GHz}$ ) de la cadence de répétition $f_{\text {rep }}=250 \mathrm{MHz}$. La détection des trains d'impulsions est assurée par une photodiode pin InGaAs. Seule la valeur absolue du coefficient est indiquée ici, la valeur réelle alterne en réalité entre valeurs positives et négatives, elle passe donc par des points «magiques » où elle s'annule.

La seconde limitation, le faible rapport signal à bruit, est liée aux effets de saturation de la photodiode de détection du train d'impulsions. À puissance optique incidente croissante, la puissance micro-onde dans une harmonique donnée augmente globalement, et l'on pourrait donc croire qu' augmenter cette puissance optique permet d'améliorer le rapport signal à bruit micro-onde. Néanmoins par effet de saturation, cela n'est pas le cas et, rapidement, l'amplitude du signal micro-onde reste constante alors que le niveau de bruit (généré par bruit thermique et bruit de grenaille) reste également constant, voire augmente à forte puissance optique incidente. Notre stratégie pour contrer cet effet s'est développée dans deux directions complémentaires. En premier lieu, l'utilisation de photodiodes à haute linéarité (Discovery Semiconductor HLPD DSC4OS) permet d'obtenir une puissance microonde supérieure si l'on dispose d'une puissance optique suffisante. Pour des cadences de répétition de $250 \mathrm{MHz}$, nous pouvons ainsi obtenir jusqu'à $-20 \mathrm{dBm}$ de puissance à $10 \mathrm{GHz}$ (des détecteurs plus standards n'atteignent au mieux qu'environ $-30 \mathrm{dBm}$ ). Une seconde approche consiste à multiplier la cadence de répétition du peigne de fréquences, afin de mettre plus d'énergie dans l'harmonique voulue (typiquement $10 \mathrm{GHz}$ ) et de supprimer les harmoniques « inutiles ». Si la cadence des oscillateurs même est très difficile à augmenter du fait des technologies utilisées pour les peignes à fibre dopée à l'erbium, des multiplicateurs de cadence externes permettent néanmoins de gagner en cadence «effective» du train d'impulsions. Le SYRTE a développé une technique originale et novatrice dans cette optique, basée sur une série d'interferomètres de Mach-Zehnder en cascade (fig. 7), qui a permis d'obtenir des cadences effectives jusqu'à $2 \mathrm{GHz}$ et des puissances micro-ondes à $10 \mathrm{GHz}$ d'environ $0 \mathrm{dBm}$ [24] (fig. 8).

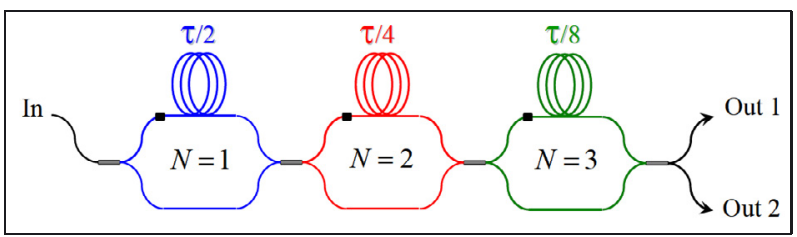

Fig. 7. - Schéma de principe d'un multiplicateur de cadence de répétition (ici à 3 étages donc multiplicateur de cadence par $2^{3}=8$ ) réalisé par cascade successives d'interféromètres de Mach-Zehnder. Les lignes à retard des trois étages sont paramétrisées par le facteur $t$, délai entre deux impulsions optiques successives en entrée, qui est adapté finement à la cadence de répétition du laser femtoseconde source.

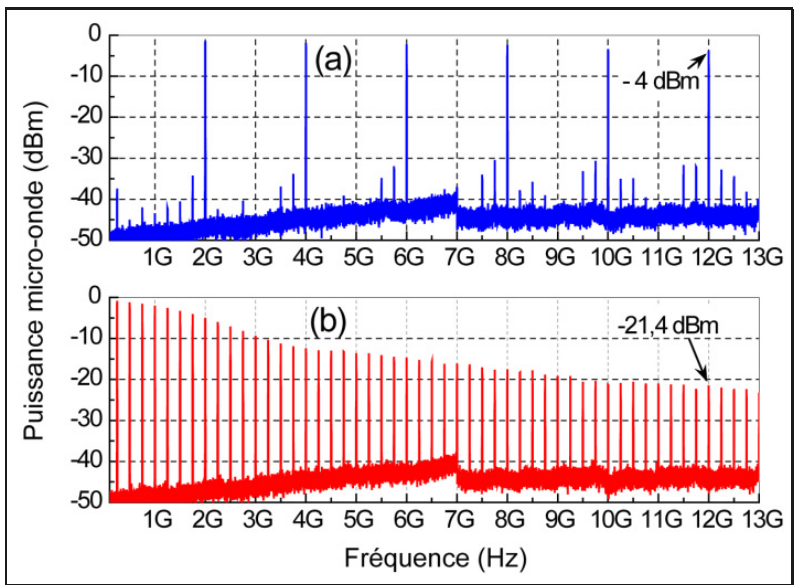

Fig. 8. - Puissance micro-onde générée par photodétection du train d'impulsions d'un laser à $250 \mathrm{MHz}$ de cadence de répétition en présence (a) et en absence (b) d'un multiplicateur de cadence de répétition fibré à base d'interféromètres de MachZehnder en cascade.

À l'issue de nos études, nous avons démontré que l'on peut limiter les bruits de phase résiduels à un niveau de $-120 \mathrm{dBc} \cdot \mathrm{Hz}^{-1}$ à $1 \mathrm{~Hz}$ d'une porteuse d'environ $10 \mathrm{GHz}$ [21], et des paliers de bruit de l'ordre $\mathrm{de}-165 \mathrm{dBc} \cdot \mathrm{Hz}^{-1}$ [24] (en extraction micro-onde à partir d'un peigne unique; en génération absolue, des travaux de caractérisation doivent encore être menés à bien pour atteindre de telles performances). Ces résultats indiquent que les systèmes de génération micro-onde par méthode photonique du SYRTE sont proches de l'état de l'art, toute technologie confondue. Le potentiel d'amélioration semble très prometteur et dresse la perspective de transfert vers l'industrie. Notons également que si des développements à des niveaux de performances similaires ont également été réalisés au NIST à l'aide de peignes basés sur des lasers titane-saphir (voir en particulier [25] et les références incluses), seuls les types de systèmes à base de fibre dopée à l'erbium permettent aujourd'hui d'envisager sereinement un niveau de fiabilité compatible avec un dispositif opérationnel, voire commercial.

\section{Conclusion}

Cet article a présenté les travaux effectués au LNE-SYRTE sur les peignes de fréquences optiques et 
a décrit les systèmes actuellement utilisés au laboratoire. Outre les mesures de fréquences optiques proprement dites, un travail important a été effectué sur la génération de signaux micro-ondes à très bas bruit de phase par transfert de la pureté spectrale d'une référence optique vers le domaine micro-onde à l'aide de ces outils. D'autres travaux visant à améliorer ces performances audelà de l'état de l'art mondial actuel continuent à être entrepris au laboratoire. Par ailleurs, une autre thématique intéressante que nous allons étudier dans le futur consistera à utiliser ces peignes pour transférer la pureté spectrale d'un laser de fréquence donnée vers une autre fréquence. Le développement de cette nouvelle thématique sera en effet très utile aux horloges optiques du laboratoire, puisqu'elles pourraient ainsi bénéficier rapidement et facilement des progrès à prévoir dans le domaine de la réalisation de lasers ultra-stables de très haute performance. Les lasers stabilisés sur cavité Fabry-Perot constituent actuellement en effet une limite à la stabilité des horloges à réseau optique, et plusieurs voies d'amélioration sont envisagées dans le monde et au LNE-SYRTE. Dans tous les cas, ces lasers ultra-stables de prochaine génération seront développés à une fréquence particulière, et le transfert par peigne de leur pureté spectrale aux fréquences d'intérêt métrologique pour le LNE-SYRTE (fréquences d'horloge des l'étalons de fréquences à mercure et strontium neutre) constituera donc une étape très importante dans le futur des horloges optiques.

\section{Références}

[1] Guéna J., Abgrall M., Rovera D., Laurent P., Chupin B., Lours M., SANTARElli G., Rosenbusch P., Tobar M., Li R., Gibble K., Clairon A. et Bize S., "Progress in atomic fountains at LNE-SYRTE", IEEE UFFC, 59, 2012, 391-410.

[2] DIDDAMS S.A., "The evolving optical frequency comb", J. Opt. Soc. Am. B, 27, 2010, 51-B62.

[3] NEWBURY N.R., "Searching for applications with a finetooth comb", Nat. Phot., 5, 2011, 186-188.

[4] Reichert J., NiERing M., HolzWarth R., Weitz M., UDEM T. et HÄNSCH T.W., "Phase Coherent VacuumUltraviolet to Radio Frequency Comparison with a ModeLocked Laser", Phys. Rev. Lett., 84, 2000, 3232-3235.

[5] Diddams S.A., Jones D.J., Ye J., CundifF S.T., Hall J.L., RANKA J.K., WINDELER R.S., HOLZWARTH R., UDEM T. et HANSCH T.W., "Direct link between microwave and optical frequencies with a $300 \mathrm{THz}$ femtosecond laser comb", Phys. Rev. Lett., 84, 2000, 5102-5105.

[6] Holzwarth R., Udem T., Hansch T.W., Knight J.C., WADSWORTH W.J. et RUSSEL P.S.J., "Optical frequency synthesizer for precision spectroscopy", Phys. Rev. Lett., $\mathbf{8 5}, 2000,2264-2267$.

[7] DidDAms S.A., Hollberg L., MA L.S., et RoberTsSON L., "Femtosecondlaser-based optical clockwork with instability $<6 \times 10^{-16}$ in 1s", Opt. Lett., 27, 2002, 58-60.

[8] RAmond T.M., DidDams S.A., Hollberg L. et BARTElS A., "Phase coherent link from optical to microwave frequencies by means of the broadband continuum from a 1-GHz Ti:sapphire femtosecond oscillator", Opt. Lett., 27, 2002, 1842-1844.

[9] Giorgetta F.R., Coddington I., BAUMANN E., SwanN W.C., NEWBURY N.R., "Fast high-resolution spectroscopy of dynamic continuous-wave laser sources", Nat. Phot., 4, 2010, 853-857.

[10] Zhang W., Lours M., Fischer M., Holzwarth R., SANTARElli G. et LE COQ Y., "Characterizing a fiberbased frequency comb with electro-optic modulator", IEEE Transactions on Ultrasonics, Ferroelectrics and Frequency Control, 59, 2012, 432.

[11] Lopez O., Haboucha A., Chanteau B., Chardonnet C., Amy-Klein A. et G. Santarelli G., "Ultra-stable long distance optical frequency distribution using the Internet fiber network", Opt. Exp., 20, 2012, 23518.

[12] Yi L., MeJri S., McFerran J.J., Le CoQ Y. et Bize S., "Optical lattice trapping of $199 \mathrm{Hg}$ and determination of the magic wavelength for the ultraviolet $1 \mathrm{~S} 0-3 \mathrm{P} 0$ clock transition", Phys. Rev. Lett., 106, 2011, 073005.

[13] McFerran J.J., Yi L., MeJri S., Di Manno S., Zhang W., GuÉNA J., LE COQ Y. et BIZE S., "Neutral atom frequency reference in the deep ultraviolet with fractional uncertainty $=5.7 \times 10^{-15}$ ", Phys. Rev. Lett., 108, 2012, 183004.

[14] LE TARGAT R., et al. "Realization of an optical second with lattice clocks", Nat. Comm., 4, 2013, 2109.

[15] Chanteau B., Lopez O., Zhang W., Nicolodo D., Argence B., Auguste F., AbGrall M., Chardonnet C., Santarelli G., Darquié B., Le Coq Y. et AmyKLEIN A., "Mid-infrared laser phase-locking to a remote near-infrared frequency reference for high-precision molecular spectroscopy", New J. Phys., 15, 2013, 073003.

[16] Millo J., Magalhaes D.V., Mandache C., Le Coq Y. ENGLISH E.M.L., WeSTERGAARD P.G., LODEWYCK J., Bize S., Lemonde P. et Santarelli G., "Ultra-stable lasers based on vibration insensitive cavities", Phys. Rev. A, 79, 5, 2009, 053829.

[17] Argence B., Prevost E., LévèQue T., Le Goff R., Bize S., LEMONDE P. et SANTARELli G. "Prototype of an ultra-stable optical cavity for space application", Opt. Exp., 20, 2012, 25409.

[18] Millo J., AbGrall M., Lours M., English E.M.L., Jiang H., GuÉna J., Clairon A., Tobar M.E., Bize S., LE COQ Y. et SANTARELLI G., "Ultralow noise microwave generation with fiber-based optical frequency comb and application to atomic fountain clock", Appl. Phys. Lett., 94, 2009, 141105.

[19] WeYers S., LiPPHARdT B. et Schnatz H., "The Stability of an Optical Clock Laser Transferred to the Interrogation Oscillator for a Cs Fountain", Phys. Rev. A, 79, 2009, 031803R.

[20] Millo J., Boudot R., Lours M., Bourgeois P.Y., Luiten A.N., Le CoQ Y., Kersale Y. et SANTARelli G., "Ultra-Low Noise Microwave Extraction from FiberBased Optical Frequency Comb", Opt. Lett., 23, 34, 2009, 3707-3709. 
[21] Zhang W., Xu Z., Lours M., Boudot R., Kersalé Y., SANTARELli G. et LE COQ Y., "Sub-100 attoseconds stability optics to microwave synchronization", Appl. Phys. Lett., 96, 2010, 211105.

[22] Zhang W., Xu Z., Lours M., Boudot R., Kersalé Y., Luiten A.N., SANTARELli G. et LE COQ Y., "Advanced noise reduction techniques for ultra-low phase noise optical-to-microwave division with femtosecond fiber combs", IEEE Transaction on Ultrasonic Ferromagnetic and Frequency Control, 58, 2011, 900.

Article reçu le 21 décembre 2012, version révisée reçue le 26 septembre 2013.
[23] Zhang W., Li T., Lours M., Seidelin S., SANTARelli G. et LE COQ Y., "Amplitude to phase conversion of InGaAs PIN photodiodes for femtosecond lasers microwave signal generation", Appl. Phys. B Lasers and Optics, 106, 2012, 301.

[24] Haboucha A., Zhang W., Li T., Lours M., Luiten A.N., Le CoQ Y. et Santarelli G., "Optical-Fiber Pulse Rate Multiplier for Ultra-low Phase-noise Signal Generation", Opt. Lett., 36, 2011, 3654.

[25] ForTIER T.M., KIRCHNER M.S., QUINLAN F., TAYLOR J. BERgQuist J.C., ROSENBAND T., LEMKE N., LUdLOW A., JiANG Y., OATES C.W. et DiDDAMS S.A., "Generation of ultra-stable microwaves via optical frequency division", Nat. Phot., 5, 7, 2011, 425-429. 